Research Article

\title{
Extrapolation Algorithm for Computing Multiple Cauchy Principal Value Integrals
}

\author{
Jin Li 1 i and Yongling Cheng $\mathbb{C}$ \\ College of Science, North China University of Science and Technology, Tangshan 063210, China \\ Correspondence should be addressed to Yongling Cheng; chengyongling1129@163.com
}

Received 22 August 2019; Revised 27 December 2019; Accepted 20 January 2020; Published 11 March 2020

Academic Editor: Gordon Huang

Copyright () 2020 Jin Li and Yongling Cheng. This is an open access article distributed under the Creative Commons Attribution License, which permits unrestricted use, distribution, and reproduction in any medium, provided the original work is properly cited.

In this paper, the computation of multiple (including two dimensional and three dimensional) Cauchy principal integral with generalized composite rectangle rule was discussed, with the density function approximated by the middle rectangle rule, while the singular kernel was analytically calculated. Based on the expansion of the density function, the asymptotic expansion formulae of error functional are obtained. A series is constructed to approach the singular point, then the extrapolation algorithm is presented, and the convergence rate is proved. At last, some numerical examples are presented to validate the theoretical analysis.

\section{Introduction}

With the development of boundary element methods, a lot of attentions had recently been paid to Cauchy principal value integrals (including one dimensional and two dimensional). In this paper, we pay our attention to certain two-dimensional Cauchy principal value integral of the form

$$
I(f, t, s)=\int_{L_{1}} \int_{L_{2}} \frac{f(x, y)}{(x-t)(y-s)} \mathrm{d} x \mathrm{~d} y,
$$

where $\int_{L_{1}} \int_{L_{2}}$ denotes a Cauchy Principle value integral $L_{1}=[a, b], L_{2}=[c, d]$, and $(t, s) \in(a, b) \times(c, d)$ the singular point.

As an adequate tool in boundary element methods, Cauchy principal value integral equations [1-7] have been appeared in many engineering problems [8], such as fluid mechanics, acoustics, fracture mechanics, elasticity, and electromagnetic scattering problems for the modeling of many physical situations. Numerous works such as the Gaussian method [9], the transformation method [10], and the Newton-Cote methods [11-15] have been devoted to developing efficient quadrature formulae. In recent years, the superconvergence phenomenon of the hypersingular integral defined on interval and circle is studied in [16-19] and the superconvergence phenomenon of the Cauchy principal value integral is also investigated in [20-22].

In [1], based on the Gauss quadrature of one-dimensional Cauchy principal value integral with weight function $\omega(x)=(1-x)^{\alpha}(l+x)^{\beta}, \alpha, \beta>-1$, generalized quadrature Gauss rule for two-dimensional Cauchy principal value integrals is presented. In [23], for the problem of computing $2 \mathrm{D}$ Cauchy principal value integrals of the form $\int_{S} F\left(P_{0}, P\right) \mathrm{d} P$, authors have constructed quadrature rules of the Gaussian type. In [3], for the two-dimensional Cauchy principal value integrals with respect to generalized smooth Jacobi weight functions, authors have considered product rules of the Gauss type for the numerical approximation of two-dimensional Cauchy principal value integrals. In [24], a principal value definition of the basic hypersingular integral in the fundamental integral equation for two-dimensional cracks in three-dimensional isotropic elasticity is proposed. In [25], the classical composite rectangle (midpoint) rule for the computation of two-dimensional singular integrals is discussed, with the error functional of the rectangle rule for computing two-dimensional singular integrals, and the local coordinate of certain point and the convergence results $O\left(h^{2}\right)$ are obtained. In [26], the classical composite trapezoidal rule for the computation of two-dimensional singular 
integrals is presented and the convergence results $O\left(h^{2}\right)$ is the same as the Riemann integral convergence rate at a certain point of the classical composite trapezoidal rule. In [27], the Cauchy principal value integral is approximated by the zeros of the Chebyshev polynomials of the first kind. In [28], a twelve-point cubature formula has been constructed for the numerical evaluation of two-dimensional real Cauchy principal value integrals. In [29], Gaussian quadrature rules for the evaluation of Cauchy principal values of integrals are considered and their relation with GaussLegendre formulae is also studied. In [30], the density function $f(x, y)$ was presented as

$$
f(x, y)-f(t, s)=f_{1}(x, y)+f_{2}(x, y)+f_{3}(x, y),
$$

where

$$
\begin{aligned}
& f_{1}(x, y)=f(x, s)-f(t, s), \\
& f_{2}(x, y)=f(t, y)-f(t, s), \\
& f_{3}(x, y)=f(x, y)-f(x, s)-f(t, y)+f(t, s) .
\end{aligned}
$$

Then, (1) can be written as

$$
\begin{aligned}
\int_{L_{1}} \int_{L_{2}} \frac{f(x, y)}{(x-t)(y-s)} \mathrm{d} x \mathrm{~d} y= & \int_{L_{1}} \frac{\mathrm{d} y}{y-s} \int_{L_{2}} \frac{f_{1}(x, y)}{x-t} \mathrm{~d} x \\
& +\int_{L_{1}} \frac{\mathrm{d} x}{x-t} \int_{L_{2}} \frac{f_{2}(x, y)}{y-s} \mathrm{~d} y \\
& +\int_{L_{1}} \int_{L_{2}} \frac{f_{3}(x, y)}{(x-t)(y-s)} \mathrm{d} x \mathrm{~d} y \\
& +f(t, s) \int_{L_{1}} \frac{\mathrm{d} x}{x-t} \int_{L_{2}} \frac{\mathrm{d} y}{y-s}
\end{aligned}
$$

which is the method with subtraction of singularity while the singular part was calculated analytically.

In $[31,32]$, Lyness studied the Euler-Maclaurin expansion technique for the evaluation of two-dimensional Cauchy principal integrals. The extrapolation methods based on Hadamard finite-part integral definition with the trapezoidal rule for the computation of hypersingular integrals on interval and in circle in boundary element methods are presented in $[33,34]$, respectively. The extrapolation methods based on definition of subtraction of singularity are presented in $[35,36]$, respectively.

In this paper, we focus on certain kinds of two-dimensional Cauchy principal value integrals which have not been extensively studied. It is the aim of this paper to investigate the error expansion of the rectangle rule and, in particular, to derive the error estimates. We examine the convergence property of the rectangle rule for certain kinds of the two-dimensional Cauchy principal value integrals and generalize the abovementioned one-dimensional convergence results to cover this new situation. Moreover, we give an error expansion of the corresponding remainder when the density function $f(x, y)$ belongs to $C^{\infty}([a, b] \times[c, d])$. As the special function of the error expansion equals zero, we get the superconvergence phenomenon, i.e., when the singular point coincides with some a priori known point, the convergence rate is higher than what is globally possible. Then, the extrapolation algorithm is presented and the convergence rate of an extrapolation error and a posterior error is proved.

The rest of this paper is organized as follows. In Section 2, after introducing some basic formulae of the rectangle rule, we present the main results. In Section 3, we finished the proof. Finally, several numerical examples are provided to validate our analysis.

\section{Main Result}

Let $a=x_{0}<x_{1}<\cdots<x_{n-1}<x_{n}=b$ and $c=y_{0}<y_{1}<\cdots<$ $y_{m-1}<y_{m}=d$ be a uniform partition of the area $[a, b] \times$ $[c, d]$ with mesh size $h_{x}=(b-a) / n$ and $h_{y}=(d-c) / m$. In order to simplify our analysis in the following, we set $h=h_{x}=h_{y}=(b-a) / n=(d-c) / n$; it is not difficult to extend our analysis to the case of quasi-uniform meshes.

We set $\widehat{x}_{i}=x_{i}+h / 2, \hat{y}_{j}=y_{j}+h / 2, i, j=0,1, \ldots, n-1$, and define $f_{C}(x, y)$ as the piecewise constant interpolation for $f(x, y)$ :

$$
f_{C}(x, y)=f\left(\hat{x}_{i}, \hat{y}_{j}\right), \quad i, j=0,1, \ldots, n-1,
$$

and then we obtain

$$
\begin{aligned}
I_{n}(f, t, s) & =\int_{a}^{b} \int_{c}^{d} \frac{f_{C}(x, y)}{(x-t)(y-s)} \mathrm{d} x \mathrm{~d} y \\
& =\sum_{i=0}^{n-1} \sum_{j=0}^{n-1} f\left(\hat{x}_{i}, \hat{y}_{j}\right) \int_{x_{i}}^{x_{i+1}} \int_{y_{j}}^{y_{j+1}} \frac{\mathrm{d} x \mathrm{~d} y}{(x-t)(y-s)} \\
& =\sum_{i=0}^{n-1} \sum_{j=0}^{n-1} f\left(\widehat{x}_{i}, \widehat{y}_{j}\right) \omega_{i, j}(t, s) \\
& =I(f, t, s)-E_{n}(f, t, s),
\end{aligned}
$$

where $E_{n}(f, t, s)$ denotes the error functional and the Cotes coefficient is

$$
\begin{aligned}
\omega_{i, j}(t, s) & =\int_{x_{i}}^{x_{i+1}} \int_{y_{j}}^{y_{j+1}} \frac{\mathrm{d} x \mathrm{~d} y}{(x-t)(y-s)} \\
& =\int_{x_{i}}^{x_{i+1}} \frac{\mathrm{d} x}{x-t} \int_{y_{j}}^{y_{j+1}} \frac{\mathrm{d} y}{y-s}=\log \left|\frac{x_{i+1}-t}{x_{i}-t}\right| \log \left|\frac{y_{j+1}-s}{y_{j}-s}\right| .
\end{aligned}
$$

Define the following linear transformation:

$$
\begin{aligned}
& x=\hat{x}_{i}(\tau):=\frac{(\tau+1)\left(x_{i+1}-x_{i}\right)}{2}+x_{i}, \quad \tau \in[-1,1], \\
& y=\hat{y}_{j}(\tau):=\frac{(\xi+1)\left(y_{j+1}-y_{j}\right)}{2}+y_{j}, \quad \xi \in[-1,1],
\end{aligned}
$$

from the reference element $[-1,1]$ to the subintervals $\left[x_{i}, x_{i+1}\right]$ and $\left[y_{j}, y_{j+1}\right]$. 
Let

$$
\phi_{\tau}(t, s)= \begin{cases}-\frac{1}{2} \int_{-1}^{1} \int_{-1}^{1} \frac{\tau}{(\tau-t)(\xi-s)} \mathrm{d} \tau \mathrm{d} \xi, & |t|<1,|s|<1, \\ -\frac{1}{2} \int_{-1}^{1} \int_{-1}^{1} \frac{\tau}{(\tau-t)(\xi-s)} \mathrm{d} \tau \mathrm{d} \xi, & |t|>1,|s|>1,\end{cases}
$$

$$
\phi_{\xi}(t, s)= \begin{cases}-\frac{1}{2} \int_{-1}^{1} \int_{-1}^{1} \frac{\xi}{(\tau-t)(\xi-s)} \mathrm{d} \tau \mathrm{d} \xi, & |t|<1,|s|<1, \\ -\frac{1}{2} \int_{-1}^{1} \int_{-1}^{1} \frac{\xi}{(\tau-t)(\xi-s)} \mathrm{d} \tau \mathrm{d} \xi, & |t|>1,|s|>1,\end{cases}
$$

which can be expressed as the Legendre function of second kinds in [37]. We also set $J:=\left\{(x, y) \in R^{2}:|x| \neq 1\right.$ and $\left.|y| \neq 1\right\}$, and the operator $W: C(J) \longrightarrow C(-1,1) \times(-1,1)$ can be defined by

$$
\begin{aligned}
W f(\tau, \xi):= & f(\tau, \xi)+\sum_{i=1}^{\infty} \sum_{j=1}^{\infty} f(2 i+\tau, 2 j+\xi) \\
& +\sum_{i=1}^{\infty} \sum_{j=1}^{\infty} f(-2 i+\tau,-2 j+\xi) .
\end{aligned}
$$

Then, we have

$$
\begin{aligned}
S_{0 x}(\tau, \xi):= & \phi_{x}(\tau, \xi)+\sum_{i=1}^{\infty} \sum_{j=1}^{\infty} \phi_{x}(2 i+\tau, 2 j+\tau) \\
& +\sum_{i=1}^{\infty} \sum_{j=1}^{\infty} \phi_{x}(-2 i+\tau,-2 j+\tau), \\
S_{0 y}(\tau, \xi):= & \phi_{y}(\tau, \xi)+\sum_{i=1}^{\infty} \sum_{j=1}^{\infty} \phi_{y}(2 i+\tau, 2 j+\tau) \\
& +\sum_{i=1}^{\infty} \sum_{j=1}^{\infty} \phi_{y}(-2 i+\tau,-2 j+\tau) .
\end{aligned}
$$

The superconvergence results of constant rectangle rules are given in the following.

Theorem 1 (Theorem 2.2 in [20]). Let $S_{0 x}(\tau, \xi)$ and $S_{0 y}(\tau, \xi)$ be defined by (12) and (13), respectively. Assume that $f(x, y) \in C^{2}[a, b] \times[c, d]$ and $t \neq x_{i}, s \neq y_{j}$, for any $i, j=0,1, \ldots, n$. For the middle rectangle rule (6), there exists a positive constant $C$, independent of $h, t$, and s, such that

$$
\begin{aligned}
E_{n}(f, t, s)= & f_{y}(t, s) S_{0 x}(\tau, \xi) h+f_{x}(t, s) S_{0 y}(\tau, \xi) h \\
& +\mathscr{R}_{n}(t, s),
\end{aligned}
$$

where $t=x_{k}+((1+\tau) / 2) h$ and $s=y_{l}+((1+\tau) / 2) h k, l=0,1$, $\ldots, n-1, \tau \in(-1,1)$.

$$
\left|\mathscr{R}_{n}(t, s)\right| \leq C\left[(|\ln h|+|\ln \gamma(\tau)|)^{2}+|\ln h|(\eta(t)+\eta(s))\right] h^{2},
$$

where

$$
\gamma(\tau)=\gamma(\xi)=\min _{0 \leq i \leq n-1} \frac{\left|t-x_{i}\right|}{h}=\min _{0 \leq j \leq n-1} \frac{\left|s-y_{j}\right|}{h}=\frac{1-|\tau|}{2},
$$

$$
\begin{aligned}
& \eta(t)=\frac{1}{(t-a)(b-t)}, \\
& \eta(s)=\frac{1}{(s-c)(d-s)}
\end{aligned}
$$

From Theorem 1, when the special functions $S_{0 x}(\tau, \xi)=S_{0 y}(\tau, \xi)=0$, we get the superconvergence phenomenon. One can see that the superconvergence rate of the (composite) rectangle rule at certain points is one order higher than their global convergence rate which is the same as the Riemann integral.

In the following theorem, we present the asymptotic expansion formulae of the error functional. The proof of this theorem will be given in the next part.

Theorem 2. Assume that $f(x, y) \in C^{\infty}[a, b] \times[c, d]$. For the rectangle rule $I_{n}(f, t, s)$ defined in (2.2), there exist certain constant $a_{i, j}(\tau, \xi)$, independent of $h, t$, and $s$, such that

$$
E_{n}(f, t, s)=\sum_{\mathbf{l}=1}^{\infty} \frac{h^{\mathbf{l}}}{2^{\mathbf{l}-1}} \sum_{i, j=0,1, \ldots, \mathbf{l}}^{i+j=\mathbf{l}} f^{(i, j)}(t, s) a_{i, j}(\tau, \xi), \quad \mathbf{l} \geq 1,
$$

where $t=x_{k}+((1+\tau) / 2) h$ and $s=y_{l}+((1+\xi) / 2) h, k, l=$ $0,1, \ldots, n-1, \tau, \xi \in(-1,1)$.

\section{Proof of Theorem 2}

In this section, we study the asymptotic expansion of the composite rectangle rule for the multiple (two dimensional) Cauchy principle value integrals.

3.1. Preliminaries. In the following analysis, $C$ will denote a generic constant that is independent of $h$ and $s$, and it may have different values in different places.

Lemma 1. Assume $(t, s) \in\left(x_{k}, x_{k+1}\right) \times\left(y_{l}, y_{l+1}\right)$ and let $a_{i}=$ $2\left(t-x_{i}\right) / h-1, b_{j}=2\left(s-y_{j}\right) / h-1,0 \leq i, j \leq n-1$. Then, we have

$$
\phi_{x}\left(a_{i}, b_{j}\right)=\left\{\begin{array}{l}
-\frac{1}{h} \int_{x_{i}}^{x_{i+1}} \int_{y_{j}}^{y_{j+1}} \frac{x-\hat{x}_{i}}{(x-t)(y-s)} \mathrm{d} x \mathrm{~d} y, \quad i=k, j=l, \\
-\frac{1}{h} \int_{x_{i}}^{x_{i+1}} \int_{y_{j}}^{y_{j+1}} \frac{x-\hat{x}_{i}}{(x-t)(y-s)} \mathrm{d} x \mathrm{~d} y, \quad i \neq k, j \neq l,
\end{array}\right.
$$


$\phi_{y}\left(a_{i}, b_{j}\right)=\left\{\begin{array}{l}-\frac{1}{h} \int_{x_{i}}^{x_{i+1}} \int_{y_{j}}^{y_{j+1}} \frac{y-\widehat{y}_{j}}{(x-t)(y-s)} \mathrm{d} x \mathrm{~d} y, \quad i=k, j=l, \\ -\frac{1}{h} \int_{x_{i}}^{x_{i+1}} \int_{y_{j}}^{y_{j+1}} \frac{y-\widehat{y}_{j}}{(x-t)(y-s)} \mathrm{d} x \mathrm{~d} y, \quad i \neq k, j \neq l .\end{array}\right.$

Proof. In the case of $i=k$ and $j=l$, we have

$$
\begin{aligned}
& \int_{x_{i}}^{x_{i+1}} \int_{y_{j}}^{y_{j+1}} \frac{x-\hat{x}_{i}}{(x-t)(y-s)} \mathrm{d} x \mathrm{~d} y \\
& =\int_{x_{i}}^{x_{i+1}} \frac{x-\hat{x}_{i}}{x-t} \mathrm{~d} x \int_{y_{j}}^{y_{j+1}} \frac{1}{y-s} \mathrm{~d} y \\
& =\lim _{\varepsilon \longrightarrow 0}\left\{\left(\int_{x_{i}}^{t-\varepsilon}+\int_{t+\varepsilon}^{x_{i+1}}\right) \frac{x-\hat{x}_{i}}{x-t} \mathrm{~d} x\right\} \lim _{\varepsilon \longrightarrow 0}\left\{\left(\int_{y_{j}}^{s-\varepsilon}+\int_{s+\varepsilon}^{y_{j+1}}\right) \frac{1}{y-s} \mathrm{~d} y\right\} \\
& =\frac{h}{2} \lim _{\varepsilon \longrightarrow 0}\left\{\left(\int_{-1}^{a_{i}-(2 \varepsilon / h)}+\int_{a_{i}+(2 \varepsilon / h)}^{1}\right) \frac{\tau}{\tau-a_{i}} \mathrm{~d} \tau\right\} \lim _{\varepsilon \longrightarrow 0}\left\{\left(\int_{-1}^{b_{j}-(2 \varepsilon / h)}+\int_{b_{j}+(2 \varepsilon / h)}^{1}\right) \frac{1}{\xi-b_{j}} \mathrm{~d} \xi\right\} \\
& =\frac{h}{2} \int_{-1}^{1} \int_{-1}^{1} \frac{\tau}{\left(\tau-a_{i}\right)\left(\xi-b_{j}\right)} \mathrm{d} \tau \mathrm{d} \xi \\
& =-h \phi_{x}\left(a_{i}, b_{j}\right) .
\end{aligned}
$$

Then, we prove the first identity in (19). The second

Proof of Theorem 2. By Taylor expansion, taking $f_{C}(x, y)$ identity in (19) can be obtained by applying the approach to the correspondent Riemann integral. The two identities in (20) can be obtained similarly as (19).

$$
\begin{aligned}
\sum_{i=0, i \neq k}^{n-1} & \sum_{j=0, j \neq l}^{n-1} \int_{x_{i}}^{x_{i+1}} \int_{y_{j}}^{y_{j+1}} \frac{f(x, y)-f_{C}(x, y)}{(x-t)(y-s)} \mathrm{d} x \mathrm{~d} y \\
= & -\sum_{i=0, i \neq k}^{n-1} \sum_{j=0, j \neq l}^{n-1} \int_{x_{i}}^{x_{i+1}} \int_{y_{j}}^{y_{j+1}} \frac{\left(\widehat{x}_{i}-x\right) f_{x}(x, y)+\left(\hat{y}_{j}-y\right) f_{y}(x, y)}{(x-t)(y-s)} \mathrm{d} x \mathrm{~d} y \\
& -\sum_{i=0, i \neq k}^{n-1} \sum_{j=0, j \neq l}^{n-1} \int_{x_{i}}^{x_{i+1}} \int_{y_{j}}^{y_{j+1}} \frac{\left(\hat{x}_{i}-x\right)^{2} f_{x x}(x, y)+\left(\widehat{y}_{j}-y\right)^{2} f_{y y}(x, y)+2\left(\widehat{x}_{i}-x\right)\left(\widehat{y}_{j}-y\right) f_{x y}(x, y)}{2 !(x-t)(y-s)} \mathrm{d} x \mathrm{~d} y \\
& -\sum_{i=0, i \neq k}^{n-1} \sum_{j=0, j \neq l}^{n-1} \int_{x_{i}}^{x_{i+1}} \int_{y_{j}}^{y_{j+1}} \frac{\sum_{p=0}^{3} C_{3}^{p}\left(\hat{x}_{i}-x\right)^{p}\left(\hat{y}_{j}-y\right)^{3-p}\left(\partial^{3} / \partial x^{p} \partial y^{3-p}\right) f(x, y)}{3 !(x-t)(y-s)} \mathrm{d} x \mathrm{~d} y-\cdots \\
& -\sum_{i=0, i \neq k}^{n-1} \sum_{j=0, j \neq l}^{n-1} \int_{x_{i}}^{x_{i+1}} \int_{y_{j}}^{y_{j+1}} \frac{\sum_{p=0}^{m} C_{m}^{p}\left(\hat{x}_{i}-x\right)^{p}\left(\hat{y}_{j}-y\right)^{m-p}\left(\partial^{m} / \partial x^{p} \partial y^{m-p}\right) f(x, y)}{m !(x-t)(y-s)} \mathrm{d} x \mathrm{~d} y-\cdots
\end{aligned}
$$


For the case $i=k$ and $j=l$, we have

$$
\begin{aligned}
& \int_{x_{k}}^{x_{k+1}} \int_{y_{l}}^{y_{l+1}} \frac{f(x, y)-f_{C}(x, y)}{(x-t)(y-s)} \mathrm{d} x \mathrm{~d} y=-\int_{x_{k}}^{x_{k+1}} \int_{y_{l}}^{y_{l+1}} \frac{\left(\widehat{x}_{k}-x\right) f_{x}(x, y)+\left(\hat{y}_{l}-y\right) f_{y}(x, y)}{(x-t)(y-s)} \mathrm{d} x \mathrm{~d} y \\
& -\int_{x_{k}}^{x_{k+1}} \int_{y_{l}}^{y_{l+1}} \frac{\left(\hat{x}_{k}-x\right)^{2} f_{x x}(x, y)+\left(\hat{y}_{l}-y\right)^{2} f_{y y}(x, y)+2\left(\hat{x}_{k}-x\right)\left(\hat{y}_{l}-y\right) f_{x y}(x, y)}{2 !(x-t)(y-s)} \mathrm{d} x \mathrm{~d} y \\
& -\int_{x_{k}}^{x_{k+1}} \int_{y_{l}}^{y_{l+1}} \frac{\sum_{p=0}^{3} C_{3}^{p}\left(\hat{x}_{k}-x\right)^{p}\left(\hat{y}_{l}-y\right)^{3-p}\left(\partial^{3} / \partial x^{p} \partial y^{3-p}\right) f(x, y)}{3 !(x-t)(y-s)} \mathrm{d} x \mathrm{~d} y-\cdots \\
& -\int_{x_{k}}^{x_{k+1}} \int_{y_{l}}^{y_{l+1}} \frac{\sum_{p=0}^{m} C_{m}^{p}\left(\hat{x}_{k}-x\right)^{p}\left(\hat{y}_{l}-y\right)^{m-p}\left(\partial^{m} / \partial x^{p} \partial y^{m-p}\right) f(x, y)}{m !(x-t)(y-s)} \mathrm{d} x \mathrm{~d} y-\cdots .
\end{aligned}
$$

Putting (22) and (23) together, we have

$$
\begin{aligned}
& \int_{a}^{b} \int_{c}^{d} \frac{f(x, y)}{(x-t)(y-s)} \mathrm{d} x \mathrm{~d} y-\sum_{i=0}^{n-1} \sum_{j=0}^{n-1} \int_{x_{i}}^{x_{i+1}} \int_{y_{j}}^{y_{j+1}} \frac{f_{C}(x, y)}{(x-t)(y-s)} \mathrm{d} x \mathrm{~d} y \\
&= \sum_{i=0}^{n-1} \sum_{j=0}^{n-1} \int_{x_{i}}^{x_{i+1}} \int_{y_{j}}^{y_{j+1}} \frac{f(x, y)}{(x-t)(y-s)} \mathrm{d} x \mathrm{~d} y-\sum_{i=0}^{n-1} \sum_{j=0}^{n-1} \int_{x_{k}}^{x_{k+1}} \int_{y_{l}}^{y_{l+1}} \frac{f_{C}(x, y)}{(x-t)(y-s)} \mathrm{d} x \mathrm{~d} y \\
&= \sum_{i=0}^{n-1} \sum_{j=0}^{n-1} \int_{x_{i}}^{x_{i+1}} \int_{y_{j}}^{y_{j+1}} \frac{f(x, y)-f_{C}(x, y)}{(x-t)(y-s)} \mathrm{d} x \mathrm{~d} y \\
&=-\sum_{i=0}^{n-1} \sum_{j=0}^{n-1} \int_{x_{i}}^{x_{i+1}} \int_{y_{j}}^{y_{j+1}} \frac{\left(\widehat{x}_{i}-x\right) f_{x}(x, y)+\left(\hat{y}_{j}-y\right) f_{y}(x, y)}{(x-t)(y-s)} \mathrm{d} x \mathrm{~d} y \\
&-\sum_{i=0}^{n-1} \sum_{j=0}^{n-1} \int_{x_{i}}^{x_{i+1}} \int_{y_{j}}^{y_{j+1}} \frac{\left(\hat{x}_{i}-x\right)^{2} f_{x x}(x, y)+\left(\hat{y}_{j}-y\right)^{2} f_{y y}(x, y)+2\left(\hat{x}_{i}-x\right)\left(\hat{y}_{j}-y\right) f_{x y}(x, y)}{2 !(x-t)(y-s)} \mathrm{d} x \mathrm{~d} y \\
&-\sum_{i=0}^{n-1} \sum_{j=0}^{n-1} \int_{x_{i}}^{x_{i+1}} \int_{y_{j}}^{y_{j+1}} \frac{\sum_{p=0}^{3} C_{3}^{p}\left(\hat{x}_{i}-x\right)^{p}\left(\hat{y}_{j}-y\right)^{3-p}\left(\partial^{3} / \partial x^{p} \partial y^{3-p}\right) f(x, y)}{3 !(x-t)(y-s)} \mathrm{d} x \mathrm{~d} y-\cdots \\
&-\sum_{i=0}^{n-1} \sum_{j=0}^{n-1} \int_{x_{i}}^{x_{i+1}} \int_{y_{j}}^{y_{j+1}} \frac{\sum_{p=0}^{m} C_{m}^{p}\left(\hat{x}_{i}-x\right)^{p}\left(\hat{y}_{j}-y\right)^{m-p}\left(\partial^{m} / \partial x^{p} \partial y^{m-p}\right) f(x, y)}{m !(x-t)(y-s)} \mathrm{d} x \mathrm{~d} y-\cdots \\
&= f_{x}(t, s) \sum_{i=0}^{n-1} \sum_{j=0}^{n-1} \int_{x_{i}}^{x_{i+1}} \int_{y_{j}}^{y_{j+1}} \frac{y-\hat{y}_{j}}{(x-t)(y-s)} \mathrm{d} x \mathrm{~d} y \\
&+\sum_{i=0}^{n-1} \sum_{j=0}^{n-1} \int_{x_{i}}^{x_{i+1}} \int_{y_{j}}^{y_{j+1}} \frac{\left(\hat{x}_{i}-x\right)^{2} f_{x x}(t, s)+\left(\hat{y}_{j}-y\right)^{2} f_{y y}(t, s)+2\left(\hat{x}_{i}-x\right)\left(\hat{y}_{j}-y\right) f_{x y}(t, s)}{2 !(x-t)(y-s)} \mathrm{d} x \mathrm{~d} y \\
& \sum_{i=0}^{n-1} \sum_{j=0}^{n-1} \int_{x_{i}}^{x_{i+1}} \int_{y_{j}}^{y_{j+1}} \frac{x-\widehat{x}_{i}}{(x-t)(y-s)} \mathrm{d} x \mathrm{~d} y
\end{aligned}
$$


$-\sum_{i=0}^{n-1} \sum_{j=0}^{n-1} \int_{x_{i}}^{x_{i+1}} \int_{y_{j}}^{y_{j+1}} \frac{\sum_{p=0}^{3} C_{3}^{p}\left(\widehat{x}_{i}-x\right)^{p}\left(\hat{y}_{j}-y\right)^{3-p}\left(\partial^{3} / \partial x^{p} \partial y^{3-p}\right) f(x, y)}{3 !(x-t)(y-s)} \mathrm{d} x \mathrm{~d} y-\cdots$

$-\sum_{i=0}^{n-1} \sum_{j=0}^{n-1} \int_{x_{i}}^{x_{i+1}} \int_{y_{j}}^{y_{j+1}} \frac{\sum_{p=0}^{m} C_{m}^{p}\left(\hat{x}_{i}-x\right)^{p}\left(\hat{y}_{j}-y\right)^{m-p}\left(\partial^{m} / \partial x^{p} \partial y^{m-p}\right) f(x, y)}{m !(x-t)(y-s)} \mathrm{d} x \mathrm{~d} y-\cdots$

$-\sum_{l=1}^{\infty} \sum_{i=0}^{n-1} \sum_{j=0}^{n-1} \int_{x_{i}}^{x_{i+1}} \int_{y_{j}}^{y_{j+1}} \frac{[(x-t)(\partial / \partial x)+(y-s)(\partial / \partial x)]^{l} f_{x}(t, s)}{l !} \frac{y-\hat{y}_{j}}{(x-t)(y-s)} \mathrm{d} x \mathrm{~d} y$

$-\sum_{l=1}^{\infty} \sum_{i=0}^{n-1} \sum_{j=0}^{n-1} \int_{x_{i}}^{x_{i+1}} \int_{y_{j}}^{y_{j+1}} \frac{[(x-t)(\partial / \partial x)+(y-s)(\partial / \partial x)]^{l} f_{y}(t, s)}{l !} \frac{x-\widehat{x}_{i}}{(x-t)(y-s)} \mathrm{d} x \mathrm{~d} y$

$-\sum_{l=1}^{\infty} \sum_{i=0}^{n-1} \sum_{j=0}^{n-1} \int_{x_{i}}^{x_{i+1}} \int_{y_{j}}^{y_{j+1}} \frac{[(x-t)(\partial / \partial x)+(y-s)(\partial / \partial x)]^{l} f_{x x}(t, s)}{l !} \frac{\left(\widehat{x}_{i}-x\right)^{2}}{2 !(x-t)(y-s)} \mathrm{d} x \mathrm{~d} y$

$-\sum_{l=1}^{\infty} \sum_{i=0}^{n-1} \sum_{j=0}^{n-1} \int_{x_{i}}^{x_{i+1}} \int_{y_{j}}^{y_{j+1}} \frac{[(x-t)(\partial / \partial x)+(y-s)(\partial / \partial x)]^{l} f_{y y}(t, s)}{l !} \frac{\left(\hat{y}_{j}-y\right)^{2}}{2 !(x-t)(y-s)} \mathrm{d} x \mathrm{~d} y$

$-\sum_{l=1}^{\infty} \sum_{i=0}^{n-1} \sum_{j=0}^{n-1} \int_{x_{i}}^{x_{i+1}} \int_{y_{j}}^{y_{j+1}} \frac{[(x-t)(\partial / \partial x)+(y-s)(\partial / \partial x)]^{l} f_{x y}(t, s)}{l !} \frac{2\left(\hat{x}_{i}-x\right)\left(\hat{y}_{j}-y\right)}{2 !(x-t)(y-s)} \mathrm{d} x \mathrm{~d} y$

$=f_{x}(t, s) \sum_{i=0}^{n-1} \sum_{j=0}^{n-1} \int_{x_{i}}^{x_{i+1}} \int_{y_{j}}^{y_{j+1}} \frac{y-\hat{y}_{j}}{(x-t)(y-s)} \mathrm{d} x \mathrm{~d} y$

$+f_{y}(t, s) \sum_{i=0}^{n-1} \sum_{j=0}^{n-1} \int_{x_{i}}^{x_{i+1}} \int_{y_{j}}^{y_{j+1}} \frac{x-\widehat{x}_{i}}{(x-t)(y-s)} \mathrm{d} x \mathrm{~d} y$

$-f_{x x}(t, s) \sum_{i=0}^{n-1} \sum_{j=0}^{n-1} \int_{x_{i}}^{x_{i+1}} \int_{y_{j}}^{y_{j+1}} \frac{\left(\widehat{x}_{i}-x\right)^{2}}{2 !(x-t)(y-s)} \mathrm{d} x \mathrm{~d} y$

$-f_{y y}(t, s) \sum_{i=0}^{n-1} \sum_{j=0}^{n-1} \int_{x_{i}}^{x_{i+1}} \int_{y_{j}}^{y_{j+1}} \frac{\left(\hat{y}_{j}-y\right)^{2}}{2 !(x-t)(y-s)} \mathrm{d} x \mathrm{~d} y$

$-2 f_{x y}(t, s) \sum_{i=0}^{n-1} \sum_{j=0}^{n-1} \int_{x_{i}}^{x_{i+1}} \int_{y_{j}}^{y_{j+1}} \frac{\left(\hat{x}_{i}-x\right)\left(\hat{y}_{j}-y\right)}{2 !(x-t)(y-s)} \mathrm{d} x \mathrm{~d} y$

$-\sum_{i=0}^{n-1} \sum_{j=0}^{n-1} \int_{x_{i}}^{x_{i+1}} \int_{y_{j}}^{y_{j+1}} \frac{\sum_{p=0}^{3} C_{3}^{p}\left(\widehat{x}_{i}-x\right)^{p}\left(\hat{y}_{j}-y\right)^{3-p}\left(\partial^{3} / \partial x^{p} \partial y^{3-p}\right) f(x, y)}{3 !(x-t)(y-s)} \mathrm{d} x \mathrm{~d} y-\cdots$

$-\sum_{i=0}^{n-1} \sum_{j=0}^{n-1} \int_{x_{i}}^{x_{i+1}} \int_{y_{j}}^{y_{j+1}} \frac{\sum_{p=0}^{m} C_{m}^{p}\left(\hat{x}_{i}-x\right)^{p}\left(\hat{y}_{j}-y\right)^{m-p}\left(\partial^{3} / \partial x^{p} \partial y^{m-p}\right) f(x, y)}{m !(x-t)(y-s)} \mathrm{d} x \mathrm{~d} y-\cdots$

$-\sum_{l=1}^{\infty} \sum_{i=0}^{n-1} \sum_{j=0}^{n-1} \int_{x_{i}}^{x_{i+1}} \int_{y_{j}}^{y_{j+1}} \frac{[(x-t)(\partial / \partial x)+(y-s)(\partial / \partial x)]^{l} f_{x}(t, s)}{l !} \frac{y-\hat{y}_{j}}{(x-t)(y-s)} \mathrm{d} x \mathrm{~d} y$

$-\sum_{l=1}^{\infty} \sum_{i=0}^{n-1} \sum_{j=0}^{n-1} \int_{x_{i}}^{x_{i+1}} \int_{y_{j}}^{y_{j+1}} \frac{[(x-t)(\partial / \partial x)+(y-s)(\partial / \partial x)]^{l} f_{y}(t, s)}{l !} \frac{x-\widehat{x}_{i}}{(x-t)(y-s)} \mathrm{d} x \mathrm{~d} y$

$-\sum_{l=1}^{\infty} \sum_{i=0}^{n-1} \sum_{j=0}^{n-1} \int_{x_{i}}^{x_{i+1}} \int_{y_{j}}^{y_{j+1}} \frac{[(x-t)(\partial / \partial x)+(y-s)(\partial / \partial x)]^{l} f_{x x}(t, s)}{l !} \frac{\left(\widehat{x}_{i}-x\right)^{2}}{2 !(x-t)(y-s)} \mathrm{d} x \mathrm{~d} y$ 


$$
\begin{aligned}
& -\sum_{l=1}^{\infty} \sum_{i=0}^{n-1} \sum_{j=0}^{n-1} \int_{x_{i}}^{x_{i+1}} \int_{y_{j}}^{y_{j+1}} \frac{[(x-t)(\partial / \partial x)+(y-s)(\partial / \partial x)]^{l} f_{y y}(t, s)}{l !} \frac{\left(\hat{y}_{j}-y\right)^{2}}{2 !(x-t)(y-s)} \mathrm{d} x \mathrm{~d} y \\
& -\sum_{l=1}^{\infty} \sum_{i=0}^{n-1} \sum_{j=0}^{n-1} \int_{x_{i}}^{x_{i+1}} \int_{y_{j}}^{y_{j+1}} \frac{[(x-t)(\partial / \partial x)+(y-s)(\partial / \partial x)]^{l} f_{x y}(t, s)}{l !} \frac{2\left(\hat{x}_{i}-x\right)\left(\hat{y}_{j}-y\right)}{2 !(x-t)(y-s)} \mathrm{d} x \mathrm{~d} y \\
& =f_{x}(t, s) h S_{0 x}(\tau, \xi)+f_{y}(t, s) h S_{0 y}(\tau, \xi) \\
& -\frac{1}{2} f_{x x}(t, s) h^{2} S_{x x}(\tau, \xi)-\frac{1}{2} f_{y y}(t, s) h^{2} S_{y y}(\tau, \xi)-f_{x y}(t, s) h^{2} S_{y y}(\tau, \xi) \\
& -\sum_{i=0}^{n-1} \sum_{j=0}^{n-1} \int_{x_{i}}^{x_{i+1}} \int_{y_{j}}^{y_{j+1}} \frac{\sum_{p=0}^{3} C_{3}^{p}\left(\hat{x}_{i}-x\right)^{p}\left(\widehat{y}_{j}-y\right)^{3-p}\left(\partial^{3} / \partial x^{p} \partial y^{3-p}\right) f(x, y)}{3 !(x-t)(y-s)} \mathrm{d} x \mathrm{~d} y-\cdots \\
& -\sum_{i=0}^{n-1} \sum_{j=0}^{n-1} \int_{x_{i}}^{x_{i+1}} \int_{y_{j}}^{y_{j+1}} \frac{\sum_{p=0}^{m} C_{m}^{p}\left(\widehat{x}_{i}-x\right)^{p}\left(\widehat{y}_{j}-y\right)^{m-p}\left(\partial^{m} / \partial x^{p} \partial y^{m-p}\right) f(x, y)}{m !(x-t)(y-s)} \mathrm{d} x \mathrm{~d} y-\cdots \\
& -\sum_{l=1}^{\infty} \sum_{i=0}^{n-1} \sum_{j=0}^{n-1} \int_{x_{i}}^{x_{i+1}} \int_{y_{j}}^{y_{j+1}} \frac{[(x-t)(\partial / \partial x)+(y-s)(\partial / \partial x)]^{l} f_{x}(t, s)}{l !} \frac{y-\hat{y}_{j}}{(x-t)(y-s)} \mathrm{d} x \mathrm{~d} y \\
& -\sum_{l=1}^{\infty} \sum_{i=0}^{n-1} \sum_{j=0}^{n-1} \int_{x_{i}}^{x_{i+1}} \int_{y_{j}}^{y_{j+1}} \frac{[(x-t)(\partial / \partial x)+(y-s)(\partial / \partial x)]^{l} f_{y}(t, s)}{l !} \frac{x-\hat{x}_{i}}{(x-t)(y-s)} \mathrm{d} x \mathrm{~d} y \\
& -\sum_{l=1}^{\infty} \sum_{i=0}^{n-1} \sum_{j=0}^{n-1} \int_{x_{i}}^{x_{i+1}} \int_{y_{j}}^{y_{j+1}} \frac{[(x-t)(\partial / \partial x)+(y-s)(\partial / \partial x)]^{l} f_{x x}(t, s)}{l !} \frac{\left(\hat{x}_{i}-x\right)^{2}}{2 !(x-t)(y-s)} \mathrm{d} x \mathrm{~d} y \\
& -\sum_{l=1}^{\infty} \sum_{i=0}^{n-1} \sum_{j=0}^{n-1} \int_{x_{i}}^{x_{i+1}} \int_{y_{j}}^{y_{j+1}} \frac{[(x-t)(\partial / \partial x)+(y-s)(\partial / \partial x)]^{l} f_{y y}(t, s)}{l !} \frac{\left(\hat{y}_{j}-y\right)^{2}}{2 !(x-t)(y-s)} \mathrm{d} x \mathrm{~d} y \\
& -\sum_{l=1}^{\infty} \sum_{i=0}^{n-1} \sum_{j=0}^{n-1} \int_{x_{i}}^{x_{i+1}} \int_{y_{j}}^{y_{j+1}} \frac{[(x-t)(\partial / \partial x)+(y-s)(\partial / \partial x)]^{l} f_{x y}(t, s)}{l !} \frac{2\left(\hat{x}_{i}-x\right)\left(\hat{y}_{j}-y\right)}{2 !(x-t)(y-s)} \mathrm{d} x \mathrm{~d} y \\
& =\sum_{\mathbf{l}=1}^{\infty} \frac{h^{\mathbf{l}}}{2^{\mathbf{l}-1}} \sum_{i, j=0,1, \ldots, \mathbf{l}}^{i+j=\mathbf{l}} f^{(i, j)}(t, s) a_{i, j}(\tau, \xi), \quad \mathbf{1} \geq 1,
\end{aligned}
$$

where we have used the Taylor expansion of $f(x, y)$ at the point $(t, s)$ :

$$
\begin{aligned}
& f_{x}(x, y)=f_{x}(t, s)+\sum_{l=1}^{\infty} \frac{[(x-t)(\partial / \partial x)+(y-s)(\partial / \partial x)]^{l} f_{x}(t, s)}{l !} \\
& f_{y}(x, y)=f_{y}(t, s)+\sum_{l=1}^{\infty} \frac{[(x-t)(\partial / \partial t)+(y-s)(\partial / \partial s)]^{l} f_{y}(t, s)}{l !}
\end{aligned}
$$


Here, we have

$$
\begin{aligned}
S_{0 x}(\tau, \xi) & =\frac{1}{h} \lim _{n \longrightarrow \infty}\left\{\sum_{i=0}^{n-1} \sum_{j=0}^{n-1} \int_{x_{i}}^{x_{i+1}} \int_{y_{j}}^{y_{j+1}} \frac{\left(y-\hat{y}_{j}\right)}{(x-t)(y-s)} \mathrm{d} x \mathrm{~d} y\right\} \\
& =\frac{1}{h} \lim _{n \longrightarrow \infty}\left\{\sum_{i=0}^{n-1} \sum_{j=0}^{n-1} \int_{x_{i}}^{x_{i+1}} \int_{y_{j}}^{y_{j+1}} \frac{\tau}{\left(\tau-a_{i}\right)\left(\xi-b_{j}\right)} \mathrm{d} \tau \mathrm{d} \xi\right\} \\
& =\lim _{n \longrightarrow \infty}\left\{\phi_{x}\left(a_{i}, b_{j}\right)+\sum_{i=1}^{m} \sum_{j=1}^{m} \phi_{x}(2 j+\tau, 2 j+\xi)+\sum_{i=0}^{n-m} \sum_{j=1}^{n-m} \phi_{x}(-2 j+\tau,-2 j+\xi)\right\} \\
& =\phi_{x}(\tau, \xi)+\sum_{i=1}^{\infty} \sum_{j=1}^{\infty} \phi_{x}(2 i+\tau, 2 j+\xi)+\sum_{i=1}^{\infty} \sum_{j=1}^{\infty} \phi_{x}(-2 i+\tau,-2 j+\xi) .
\end{aligned}
$$

Similarly, we can have

$$
\begin{aligned}
S_{0 y}(\tau, \xi) & =\frac{1}{h} \lim _{n \longrightarrow \infty}\left\{\sum_{i=0}^{n-1} \sum_{j=0}^{n-1} \int_{x_{i}}^{x_{i+1}} \int_{y_{j}}^{y_{j+1}} \frac{x-\widehat{x}_{i}}{(x-t)(y-s)} \mathrm{d} x \mathrm{~d} y\right\} \\
& =\phi_{y}(\tau, \xi)+\sum_{i=1}^{\infty} \sum_{j=1}^{\infty} \phi_{y}(2 i+\tau, 2 j+\xi)+\sum_{i=1}^{\infty} \sum_{j=1}^{\infty} \phi_{y}(-2 i+\tau,-2 j+\xi), \\
S_{x x}(\tau, \xi) & =\frac{1}{h^{2}} \sum_{i=0}^{n-1} \sum_{j=0}^{n-1} \int_{x_{i}}^{x_{i+1}} \int_{y_{j}}^{y_{j+1}} \frac{\left(\hat{x}_{i}-x\right)^{2}}{(x-t)(y-s)} \mathrm{d} x \mathrm{~d} y \\
& =\phi_{x x}(\tau, \xi)+\sum_{i=1}^{\infty} \sum_{j=1}^{\infty} \phi_{x x}(2 i+\tau, 2 j+\xi)+\sum_{i=1}^{\infty} \sum_{j=1}^{\infty} \phi_{x x}(-2 i+\tau,-2 j+\xi), \\
S_{y y}(\tau, \xi) & =\frac{1}{h^{2}} \sum_{i=0}^{n-1} \sum_{j=0}^{n-1} \int_{x_{i}}^{x_{i+1}} \int_{y_{j}}^{y_{j+1}} \frac{\left(\hat{y}_{j}-y\right)^{2}}{(x-t)(y-s)} \mathrm{d} x \mathrm{~d} y \\
& =\phi_{y y}(\tau, \xi)+\sum_{i=1}^{\infty} \sum_{j=1}^{\infty} \phi_{y y}(2 i+\tau, 2 j+\xi)+\sum_{i=1}^{\infty} \sum_{j=1}^{\infty} \phi_{y y}(-2 i+\tau,-2 j+\xi), \\
S_{x y}(\tau, \xi) & =\frac{1}{h^{2}} \sum_{i=0}^{n-1} \sum_{j=0}^{n-1} \int_{x y}^{x_{i+1}} \int_{x_{i}}^{y_{j+1}} \frac{\left(\hat{x}_{i}-x\right)\left(\hat{y}_{j}-y\right)}{(x-t)(y-s)} \mathrm{d} x \mathrm{~d} y \\
y_{j} & \sum_{i=1}^{\infty} \sum_{j=1}^{\infty} \phi_{x y}(2 i+\tau, 2 j+\xi)+\sum_{i=1}^{\infty} \sum_{j=1}^{\infty} \phi_{x y}(-2 i+\tau,-2 j+\xi),
\end{aligned}
$$


where

$$
\begin{aligned}
& \phi_{x x}\left(a_{i}, b_{j}\right)=\left\{\begin{array}{l}
-\frac{1}{h^{2}} \int_{x_{i}}^{x_{i+1}} \int_{y_{j}}^{y_{j+1}} \frac{\left(x-\hat{x}_{i}\right)^{2}}{(x-t)(y-s)} \mathrm{d} x \mathrm{~d} y, \quad i=k, j=l, \\
-\frac{1}{h^{2}} \int_{x_{i}}^{x_{i+1}} \int_{y_{j}}^{y_{j+1}} \frac{\left(x-\hat{x}_{i}\right)^{2}}{(x-t)(y-s)} \mathrm{d} x \mathrm{~d} y, \quad i \neq k, j \neq l,
\end{array}\right. \\
& \phi_{y y}\left(a_{i}, b_{j}\right)=\left\{\begin{array}{l}
-\frac{1}{h^{2}} \int_{x_{i}}^{x_{i+1}} \int_{y_{j}}^{y_{j+1}} \frac{\left(y-\hat{y}_{j}\right)^{2}}{(x-t)(y-s)} \mathrm{d} x \mathrm{~d} y, \quad i=k, j=l, \\
-\frac{1}{h^{2}} \int_{x_{i}}^{x_{i+1}} \int_{y_{j}}^{y_{j+1}} \frac{\left(y-\hat{y}_{j}\right)^{2}}{(x-t)(y-s)} \mathrm{d} x \mathrm{~d} y, \quad i \neq k, j \neq l,
\end{array}\right. \\
& \phi_{x y}\left(a_{i}, b_{j}\right)=\left\{\begin{array}{l}
-\frac{1}{h^{2}} \int_{x_{i}}^{x_{i+1}} \int_{y_{j}}^{y_{j+1}} \frac{\left(x-\hat{x}_{i}\right)\left(y-\hat{y}_{j}\right)}{(x-t)(y-s)} \mathrm{d} x \mathrm{~d} y, \quad i=k, j=l, \\
-\frac{1}{h^{2}} \int_{x_{i}}^{x_{i+1}} \int_{y_{j}}^{y_{j+1}} \frac{\left(x-\hat{x}_{i}\right)\left(y-\hat{y}_{j}\right)}{(x-t)(y-s)} \mathrm{d} x \mathrm{~d} y, \quad i \neq k, j \neq l .
\end{array}\right.
\end{aligned}
$$

As for the part of

$$
\sum_{i=0}^{n-1} \sum_{j=0}^{n-1} \int_{x_{i}}^{x_{i+1}} \int_{y_{j}}^{y_{j+1}}\left[\left(y-\widehat{y}_{j}\right)^{k}\left(x-\widehat{x}_{i}\right)^{l}\right] \mathrm{d} x \mathrm{~d} y,
$$

which can be considered as the error estimate of the rectangle rule for the definite integral $\int_{a}^{b} \int_{c}^{d}(x-t)^{i}(y-s)^{j} \mathrm{~d} x \mathrm{~d} y$, $i, j \geq 1$. Obviously, by the theorem ([38], Theorem D.2.6 P470), it can be expanded by the Euler-Maclaurin expansions, and we have

$$
E_{n}^{i}(f, h)=\int_{a}^{b} \int_{c}^{d}(x-t)^{i}(y-s)^{j} \mathrm{~d} x \mathrm{~d} y+\sum_{k=1}^{\infty} c_{2 k} h^{2 k} .
$$

It is easy to see that there is no relation with the singular point $(t, s)$ and the Proof of Theorem 2 is completed.

Here, we can easily get that $a_{01}(\tau, \xi)=S_{0 y}(\tau, \xi)$ and $a_{10}(\tau, \xi)=S_{0 x}(\tau, \xi)$ and $a_{0,2}(\tau, \xi)=S_{y y}(\tau, \xi), a_{2,0}(\tau, \xi)=S_{x x}$ $(\tau, \xi)$, and $a_{1,1}(\tau, \xi)=S_{x y}(\tau, \xi)+S_{y x}(\tau, \xi)$. For the onedimensional Cauchy principal integral, we get the superconvergence point as $\pm(2 / 3)$. As we have proved above, when $a_{01}(\tau, \xi)=S_{0 y}(\tau, \xi)=a_{10}(\tau, \xi)=S_{0 x}(\tau, \xi)=0$, then we get the superconvergence point of certain two-dimensional $( \pm(2 / 3), \pm(2 / 3))$ which is the same as reference [20].

It is not difficult to extend our analysis to the case of multidimensional Cauchy principal value integrals. Let $f\left(t_{1}, t_{2}, \ldots, t_{k}\right) \in C^{\infty}\left[a_{i}, b_{i}\right]^{k} a_{i}=x_{i 0}<x_{i 1}<\cdots<x_{i, n-1}<x_{i n}=b_{i}$ be a uniform partition of the area $\left[a_{i}, b_{i}\right]^{k}$ with mesh size $h_{k x}=\left(b_{k i}-a_{k i}\right) / n$. We set $\widehat{x}_{k i}=x_{k i}+h / 2, i=0,1, \ldots, n-1$, and define $f_{C}\left(x_{k 1}, x_{k 2}, \ldots, x_{k n}\right)$ as the piecewise constant interpolation for $f(x, y)$ :

$$
f_{C}\left(x_{k 1}, x_{k 2}, \ldots, x_{k n}\right)=f\left(\widehat{x}_{k 1}, \ldots, \widehat{x}_{k n}\right) .
$$

Then, we obtain

$$
\begin{aligned}
I_{n}\left(f ; t_{1}, t_{2}, \ldots, t_{k}\right) & =\int_{a_{1}}^{b_{1}} \cdots \int_{a_{k}}^{b_{k}} \frac{f_{C}\left(x_{1}, x_{2}, \ldots, x_{k}\right)}{\left(x_{1}-t_{1}\right) \cdots\left(x_{k}-t_{k}\right)} \mathrm{d} x_{1} \cdots \mathrm{d} x_{k} \\
& =\sum_{i_{1}=0}^{n-1} \cdots \sum_{i_{k}=0}^{n-1} f\left(\widehat{x}_{k 1}, \ldots, \widehat{x}_{k n}\right) \omega_{i_{1}, \ldots, i_{k}}\left(t_{1}, t_{2}, \ldots, t_{k}\right) \\
& =I\left(f ; t_{1}, t_{2}, \ldots, t_{k}\right)-E_{n}\left(f ; t_{1}, t_{2}, \ldots, t_{k}\right),
\end{aligned}
$$


where $E_{n}\left(f ; t_{1}, t_{2}, \ldots, t_{k}\right)$ denotes the error functional and the Cotes coefficient is

$$
\omega_{i_{1}, \ldots, i_{k}}\left(t_{1}, t_{2}, \ldots, t_{k}\right)=\log \left|\frac{x_{1, i+1}-t_{1}}{x_{1 i}-t_{1}}\right| \cdots \log \left|\frac{x_{k, i+1}-t_{k}}{x_{k i}-t_{k}}\right| .
$$

Theorem 3. Assume that $f\left(t_{1}, t_{2}, \ldots, t_{k}\right) \in C^{\infty}[a, b]^{k}$. For the rectangle rule $I_{n}\left(f ; t_{1}, t_{2}, \ldots, t_{k}\right)$ defined in (36), there exist certain constant $a_{i_{1}, \ldots, i_{k}}\left(\tau_{1}, \ldots, \tau_{k}\right)$, independent of $h$ and $t_{1}, t_{2}, \ldots, t_{k}$, such that

$$
\begin{aligned}
E_{n}\left(f, t_{1}, t_{2}, \ldots, t_{k}\right)= & \sum_{\mathbf{l}=1}^{\infty} \frac{h^{\mathbf{1}}}{2^{\mathbf{l}-1}} \sum_{i_{1}, \ldots, i_{k}=0,1, \ldots, 1}^{i_{1}, \ldots, i_{k}=\mathbf{1}} f^{\left(i_{1}, \ldots, i_{k}\right)}\left(t_{1}, t_{2}, \ldots, t_{k}\right) \\
& \cdot a_{i_{1}, \ldots, i_{k}}\left(\tau_{1}, \ldots, \tau_{k}\right),
\end{aligned}
$$

where $l \geq 1, t_{1}=x_{1 k}+\left(\left(1+\tau_{1}\right) / 2\right) h, \ldots, t_{k}=x_{k l}+\left(\left(1+\tau_{k}\right) / 2\right)$ $h, i_{1}, \ldots, i_{k}=0,1, \ldots, n-1$.

\section{Extrapolation Method}

In the abovementioned sections, we have proved that the error functional of the middle rectangle rule has the following asymptotic expansion:

$$
E_{n}(f, t, s)=\sum_{\mathbf{l}=1}^{\infty} \frac{h^{\mathbf{1}}}{2^{\mathbf{l}-1}} \sum_{i, j=0,1, \ldots, \mathbf{l}}^{i+j=\mathbf{l}} f^{(i, j)}(t, s) a_{i, j}(\tau, \xi), \quad \mathbf{l} \geq 1 .
$$

It is easy to see that the error functional depends on the value of $a_{i, j}(\tau, \xi)$.

Now we present an algorithm for the given $(t, s)$. Assume there exist positive integers $n_{01}$ and $n_{02}$ such that

$$
\begin{aligned}
& m_{10}:=\frac{n_{10}(t-a)}{b-a}, \\
& m_{20}:=\frac{n_{20}(s-c)}{d-c},
\end{aligned}
$$

are positive number. In order to simplify the deduction process, we assume that $n_{10}=m_{20}$. Firstly, we partition $[a, b] \times[c, d]$ into $n_{01}$ equal subinterval to get a mesh denoted by $\Pi_{1}$ with mesh size $h_{1}=(b-a) / n_{10}=(d-c) / n_{20}$. Then, we refine $\Pi_{1}$ to get mesh $\Pi_{2}$ with mesh size $h_{2}=h_{1} / 2$. In this way, we get a series of meshes $\left\{\Pi_{j}\right\}(j=1,2, \ldots)$ in which $\Pi_{j}$ is refined from $\Pi_{j-1}$ with the mesh size denoted by $h_{j}$. The extrapolation scheme is presented in Table 1 .

For a given $\tau, \xi \in(-1,1)$, define

$$
\begin{aligned}
t_{i} & =t+\frac{\tau+1}{2} h_{i}, i=1,2, \ldots, \\
s_{j} & =s+\frac{\xi+1}{2} h_{j}, j=1,2, \ldots, \\
T\left(h_{i}\right) & =T\left(h_{i}, h_{j}\right)=I_{2^{i-1} n_{10}, 2^{j-1} n_{20}}\left(f, t_{i}, s_{j}\right) .
\end{aligned}
$$

TABle 1: Extrapolation scheme of $T_{k}^{(i)}$.

\begin{tabular}{ccccc}
\hline$T\left(h_{1}\right)=T_{1}^{(1)}$ & & & & \\
$T\left(h_{2}\right)=T_{1}^{(2)}$ & $T_{2}^{(1)}$ & & & \\
$T\left(h_{3}\right)=T_{1}^{(3)}$ & $T_{2}^{(2)}$ & $T_{3}^{(1)}$ & & \\
$T\left(h_{4}\right)=T_{1}^{(4)}$ & $T_{2}^{(3)}$ & $T_{3}^{(2)}$ & $T_{4}^{(1)}$ & \\
$T\left(h_{5}\right)=T_{1}^{(5)}$ & $T_{2}^{(4)}$ & $T_{3}^{(3)}$ & $T_{4}^{(2)}$ & $T_{5}^{(1)}$ \\
$\vdots$ & $\vdots$ & $\vdots$ & $\vdots$ & $\vdots$ \\
\hline
\end{tabular}

We present the following extrapolation algorithm.

Step one:

$$
\text { Compute } T_{1}^{(j)}=T\left(h_{j}\right), \quad j=1, \ldots, m .
$$

Step two:

$$
\begin{aligned}
& \text { Compute } T_{i}^{(j)}=T_{i-1}^{(j+1)}+\frac{T_{i-1}^{(j+1)}-T_{i-1}^{(j)}}{2^{i-1}-1}, \\
& i=2, \ldots, m, j=1, \ldots, m-i .
\end{aligned}
$$

Theorem 4. Under the asymptotic expansion of Theorem 2, for a given $\tau, \xi$ and the series of meshes defined by (42), we have

$$
\left|I(f, t, s)-T_{i}^{(j)}\right| \leq C h^{i}
$$

and a posteriori asymptotic error estimate is given by

$$
\left|\frac{T_{i-1}^{(j+1)}-T_{i-1}^{(j)}}{2^{i-1}-1}\right| \leq C h^{i-1}, \quad i \geq 2 .
$$

Proof. By the asymptotic expansion of (40), for given $\tau$, we have

$$
\begin{aligned}
I(f, t, s)-T\left(h_{i}\right)= & I(f, t, s)-I\left(f, t_{i}, s_{j}\right)+I\left(f, t_{i}, s_{j}\right)-T\left(h_{i}\right) \\
= & I(f, t, s)-I\left(f, t_{i}, s_{j}\right) \\
& +\sum_{\mathbf{l}=1}^{\infty} \frac{h^{\mathbf{1}}}{2^{\mathbf{l}-1}} \sum_{i, j=0,1, \ldots, \mathbf{1}}^{i+j=\mathbf{1}} f^{(i, j)}(t, s) a_{i, j}(\tau, \xi) .
\end{aligned}
$$

By the definition of Cauchy principal integral and (42), for the first two parts of (48) by Taylor expansion for $I\left(f, t_{i}, s_{j}\right)$ at the point $(t, s)$,

$$
\begin{aligned}
I\left(f, t_{i}, s_{j}\right)= & I(f ; t, s)+\sum_{i, j=0,1}^{1} I^{(i, j)}(f, t, s)\left(\frac{\tau+1}{2} h_{i}+\frac{\xi+1}{2} h_{i}\right) \\
& +\sum_{i, j=0,1,2}^{2} I^{(i, j)}(f, t, s)\left(\frac{\tau+1}{2} h_{i}+\frac{\xi+1}{2} h_{i}\right)^{2} \\
& +\cdots+\sum_{i, j=0,1, \ldots, l}^{l} I^{(i, j)}(f, t, s)\left(\frac{\tau+1}{2} h_{i}+\frac{\xi+1}{2} h_{i}\right)^{l}+\cdots
\end{aligned}
$$


Similarly we also expand $f^{(i, j)}\left(t_{i}, s_{j}\right)$ at point $(t, s)$, and then we have

$$
\begin{aligned}
f^{(i, j)}\left(t_{i}, s_{j}\right)= & f^{(i, j)}(t, s)+\sum_{k, l=0,1,}^{1} f^{(i+k, j+l)}(t, s)\left(\frac{\tau+1}{2} h_{i}+\frac{\xi+1}{2} h_{i}\right) \\
& +\sum_{k, l=0,1,2}^{2} \frac{f^{(i+k, j+l)}(t, s)}{2 !}\left(\frac{\tau+1}{2} h_{i}+\frac{\xi+1}{2} h_{i}\right)^{2} \\
& +\cdots+\sum_{k, l=0,1, \ldots, m}^{m} \frac{f^{(i+k, j+l)}(t, s)}{(l-i-1) !}\left(\frac{\tau+1}{2} h_{i}+\frac{\xi+1}{2} h_{i}\right)^{l-i-1}+\cdots
\end{aligned}
$$

Putting (48)-(50) together, we have

$$
I(f, t, s)-T\left(h_{i}\right)=\sum_{i=1}^{\infty} b_{i}(t, s, \tau) h_{1}^{i},
$$

where

$$
\begin{aligned}
b_{i}(t, s, \tau, \xi)= & \sum_{i, j=0,1, \ldots, l}^{l} f^{(i, j)}(t, s) \sum_{k=1}^{i} \frac{a_{i, j}(\tau, \xi)}{2^{k}}\left(\frac{\tau+1}{2}+\frac{\xi+1}{2}\right)^{i-k} \frac{1}{(i-k) !} \\
& -\frac{(\tau+1)^{i}}{2^{i} i !} \sum_{i, j=0,1, \ldots, l}^{l} I^{(i, j)}(f, t, s) .
\end{aligned}
$$

Then, $b_{i}(t, s, \tau, \xi)$ is a constant for a given $\tau$ and $\xi$. By (51), we can obtain

$$
I(f, t, s)-T\left(h_{i+1}\right)=\sum_{i=1}^{\infty} b_{i}(t, s, \tau) h_{2}^{i+1}
$$
have

By equations (51) and (53), with $h_{j}=2 h_{j+1}$, we easily

$$
\begin{aligned}
I(f, t, s) & =2 T\left(h_{i+1}\right)-T\left(h_{i}\right)+\sum_{i=2}^{\infty} b_{i}(t, s, \tau)\left(\frac{1}{2^{i-1}}-1\right) h_{i}^{j} \\
& =T_{2}^{(j)}+\sum_{i=2}^{\infty} b_{i}(t, s, \tau)\left(\frac{1}{2^{i-1}}-1\right) h_{i}^{j}
\end{aligned}
$$

which means

$$
\begin{aligned}
I(f, t, s)-T_{2}^{(j)} & =\sum_{i=2}^{\infty} b_{i}(t, s, \tau)\left(\frac{1}{2^{i-1}}-1\right) h_{j}^{i}, \\
T_{2}^{(j)} & =2 T\left(h_{i+1}\right)-T\left(h_{i}\right) .
\end{aligned}
$$

Following the extrapolation process above, we can get the accuracy $O\left(h^{3}\right)$, and we continue to use extrapolation process again and get $O\left(h^{4}\right)$. In this way, we finish the proof by mathematical induction.

\section{Numerical Example}

In this section, computational results are reported to confirm our theoretical analysis.
Example 1. Consider the Cauchy principal value integral

$$
\int_{-1}^{1} \int_{-1}^{1} \frac{x^{3} y^{3}}{(x-t)(y-s)} \mathrm{d} x \mathrm{~d} y, \quad(t, s) \in(-1,1) \times(-1,1)
$$

with the exact solution

$$
\left(t^{3} \log \frac{1-t}{1+t}+2 t^{2}+\frac{2}{3}\right)\left(s^{3} \log \frac{1-s}{1+s}+2 s^{2}+\frac{2}{3}\right) .
$$

We adopt the uniform meshes to examine the convergence rate of the rectangle rule with the dynamic point with $t=x_{[n / 4]}+(1+\tau) h / 2$ and $s=y_{[n / 4]}+(\xi+1) h / 2$. From Table 2, we know that when the local coordinate of the singular point is $( \pm(2 / 3), \pm(2 / 3))$, the quadrature reaches the convergence rate of $O\left(h^{2}\right)$; as for the nonsupersingular point, the convergence rate is $O(h)$ which agree with our theoretical analysis. For the case of $t=x_{[n / 4]}+$ $(\tau+1) h / 2$ and $s=b-(\tau+1) h / 2$ and $t=a+(\tau+1) h / 2$ and $s=$ $b-(\tau+1) h / 2$, Tables 3 and 4 show that there is no superconvergence phenomenon for the midrectangle rue which coincide with our theoretical analysis.

Example 2. In this example, we still consider the Cauchy principal value integral

$$
\int_{-1}^{1} \int_{-1}^{1} \frac{x^{3} y^{6}}{(x-t)(y-s)} \mathrm{d} x \mathrm{~d} y, \quad(t, s) \in(-1,1) \times(-1,1)
$$

with the exact solution

$$
\left(t^{3} \log \frac{1-t}{1+t}+2 t^{2}+\frac{2}{3}\right)\left(2 s^{5}+\frac{2}{3} s^{3}+\frac{2}{5} s+s^{6} \log \frac{1-s}{1+s}\right) .
$$

Tables 5 and 6 show extrapolation errors of the rectangle rule with the singular points $s=x_{[n / 4]}+(\tau+1) h / 2$ and $t=y_{[n / 4]}+(\tau+1) h / 2$; the convergence rate of the rectangle rule is $O(h), O\left(h^{2}\right), O\left(h^{3}\right), \ldots$, while a posterior error of the rectangle rule extrapolation convergence rate of the rectangle rule is $O(h), O\left(h^{2}\right), O\left(h^{3}\right), \ldots$, with $\tau=\xi=0$, which agree with our Theorem 4 .

Tables 7 and 8 show extrapolation errors of the rectangle rule with the singular points $s=x_{[n / 4]}+(\tau+1) h / 2$ and $t=y_{[n / 4]}+(\tau+1) h / 2$, which show that the convergence rate of the rectangle rule is $O(h), O\left(h^{2}\right), O\left(h^{3}\right), \ldots$, while a posterior error of the rectangle rule extrapolation convergence rate of the rectangle rule is $O(h), O\left(h^{2}\right), O\left(h^{3}\right), \ldots$, with $\tau=\xi=2 / 3$, which agrees with our Theorem 4 .

Example 3. Consider the Cauchy principal value integral

$$
\begin{array}{r}
\int_{-1}^{1} \int_{-1}^{1} \int_{-1}^{1} \frac{x^{3} y^{6} z^{3}}{(x-t)(y-s)(z-u)} \mathrm{d} x \mathrm{~d} y \mathrm{~d} z, \\
(t, s, u) \in(-1,1) \times(-1,1) \times(-1,1),
\end{array}
$$

with the exact solution 
TABLE 2: Errors of the rectangle rule with $t=x_{[n / 4]}+(\tau+1) h / 2$ and $s=y_{[n / 4]}+(\xi+1) h / 2$.

\begin{tabular}{lccccc}
\hline$n$ & $(0,0)$ & $(2 / 3,2 / 3)$ & $(-2 / 3,-2 / 3)$ & $(2 / 3,-2 / 3)$ & $(-2 / 3,2 / 3)$ \\
\hline 64 & $5.8370 e-02$ & $5.1411 e-03$ & $-1.4502 e-03$ & $1.9813 e-03$ & $1.9813 e-03$ \\
128 & $3.1292 e-02$ & $1.4307 e-03$ & $-3.6303 e-04$ & $5.5081 e-04$ & $5.5081 e-04$ \\
256 & $1.6182 e-02$ & $3.7674 e-04$ & $-9.0779 e-05$ & $1.4508 e-04$ & $1.4508 e-04$ \\
512 & $8.2257 e-03$ & $9.6619 e-05$ & $-2.2695 e-05$ & $3.7224 e-05$ & $3.7224 e-05$ \\
1024 & $4.1467 e-03$ & $2.4462 e-05$ & $-5.6736 e-06$ & $9.4270 e-06$ & $9.4270 e-06$ \\
$h^{\alpha}$ & 0.954 & 1.9288 & 1.9994 & 1.9289 & 1.9289 \\
\hline
\end{tabular}

TABLE 3: Errors of the rectangle rule with $t=x_{[n / 4]}+(\tau+1) h / 2$ and $s=b-(\xi+1) h / 2$

\begin{tabular}{lccccc}
\hline$n$ & $(0,0)$ & $(2 / 3,2 / 3)$ & $(-2 / 3,-2 / 3)$ & $(2 / 3,-2 / 3)$ & $(-2 / 3,2 / 3)$ \\
\hline 64 & $-7.7845 e-02$ & $-1.8981 e-02$ & $-6.3590 e-02$ & $-6.9936 e-02$ & $-1.4985 e-02$ \\
128 & $-5.0142 e-02$ & $-9.6972 e-03$ & $-3.3326 e-02$ & $-3.5663 e-02$ & $-8.1438 e-03$ \\
256 & $-3.0714 e-02$ & $-4.8309 e-03$ & $-1.7118 e-02$ & $-1.7883 e-02$ & $-4.2928 e-03$ \\
512 & $-1.8182 e-02$ & $-2.3911 e-03$ & $-8.6906 e-03$ & $-8.9255 e-03$ & $-2.2176 e-03$ \\
1024 & $-1.0503 e-02$ & $-1.1842 e-03$ & $-4.3828 e-03$ & $-4.4522 e-03$ & $-1.1308 e-03$ \\
$h^{\alpha}$ & 0.7225 & 1.0006 & 0.9647 & 0.9934 & 0.9320 \\
\hline
\end{tabular}

TABLE 4: Errors of the rectangle rule with $t=a+(\tau+1) h / 2$ and $s=b-(\xi+1) h / 2$

\begin{tabular}{lccccc}
\hline$n$ & $(0,0)$ & $(2 / 3,2 / 3)$ & $(-2 / 3,-2 / 3)$ & $(2 / 3,-2 / 3)$ & $(-2 / 3,2 / 3)$ \\
\hline 64 & $-2.3835 e-01$ & $-2.3005 e-02$ & $-2.3005 e-02$ & $-2.3036 e-02$ & $-5.7772 e-02$ \\
128 & $-1.5647 e-01$ & $-8.5913 e-03$ & $-8.5913 e-03$ & $1.2073 e-02$ & $-4.0355 e-02$ \\
256 & $-9.8100 e-02$ & $-2.9871 e-03$ & $-2.9871 e-03$ & $1.7480 e-02$ & $-2.6800 e-02$ \\
512 & $-5.9436 e-02$ & $-9.8785 e-04$ & $-9.8785 e-04$ & $1.4112 e-02$ & $-1.7061 e-02$ \\
1024 & $-3.5070 e-02$ & $-3.1487 e-04$ & $-3.1487 e-04$ & $9.5823 e-03$ & $-1.0489 e-02$ \\
$h^{\alpha}$ & 0.6912 & 1.5478 & 1.5939 & 0.3164 & 0.6154 \\
\hline
\end{tabular}

TABLE 5: Extrapolation errors of the rectangle rule with $s=x_{[n / 4]}+(\tau+1) h / 2, t=y_{[n / 4]}+(\tau+1) h / 2$, and $\tau=\xi=0$.

\begin{tabular}{lcccc}
\hline & $h$ extra & $h^{2}-$ extra & $h^{3}-$ extra & \\
\hline 16 & $-1.1103 e-01$ & & & \\
32 & $-6.1657 e-02$ & $-1.2288 e-02$ & & \\
64 & $-3.2546 e-02$ & $-3.4355 e-03$ & $-4.8471 e-04$ & $-3.4340 e-06$ \\
128 & $-1.6726 e-02$ & $-9.0658 e-04$ & $-6.3594 e-05$ & $-1.4752 e-07$ \\
256 & $-8.4795 e-03$ & $-2.3270 e-04$ & $-8.0783 e-06$ & $-6.4608 e-09$ \\
512 & $-4.2692 e-03$ & $-5.8938 e-05$ & $-1.0154 e-06$ & $-3.0565 e-10$ \\
1024 & $-2.1420 e-03$ & $-1.4830 e-05$ & $-1.2720 e-07$ & \\
\hline
\end{tabular}

TABLE 6: A posterior error of the rectangle rule with $s=x_{[n / 4]}+(\tau+1) h / 2, t=y_{[n / 4]}+(\tau+1) h / 2$, and $\tau=\xi=0$.

\begin{tabular}{lcccc}
\hline & $h$-extra & $h^{2}-$ extra & $h^{3}-$ extra & \\
\hline 16 & & & & \\
32 & $-4.9369 e-02$ & & & \\
64 & $-2.9111 e-02$ & $-2.9508 e-03$ & $-6.0160 e-05$ & \\
128 & $-1.5820 e-02$ & $-8.4299 e-04$ & $-7.9308 e-06$ & $-2.1910 e-07$ \\
256 & $-8.2468 e-03$ & $-2.2463 e-04$ & $-1.0090 e-06$ & $-9.4039 e-09$ \\
512 & $-4.2103 e-03$ & $-5.7922 e-05$ & $-1.2689 e-07$ & $-4.1034 e-10$ \\
1024 & $-2.1272 e-03$ & $-1.4703 e-05$ & & \\
\hline
\end{tabular}

$$
\begin{aligned}
& \left(t^{3} \log \frac{1-t}{1+t}+2 t^{2}+\frac{2}{3}\right)\left(2 s^{5}+\frac{2}{3} s^{3}+\frac{2}{5} s+s^{6} \log \frac{1-s}{1+s}\right) \\
& \cdot\left(u^{3} \log \frac{1-u}{1+u}+2 u^{2}+\frac{2}{3}\right) .
\end{aligned}
$$

Tables 9 and 10 show extrapolation errors of the rectangle rule with the singular points $t=x_{[n / 4]}+\left(\tau_{1}+1\right) h / 2$, $s=x_{[n / 4]}+\quad\left(\tau_{2}+1\right) h / 2$, and $u=x_{[n / 4]}+\left(\tau_{3}+1\right) h / 2 ; \quad$ the convergence rate of rectangle rule is $O(h), O\left(h^{2}\right), O\left(h^{3}\right), \ldots$, for the general convergence rate, while a posterior error of 
TABLE 7: Extrapolation errors of the rectangle rule with $s=x_{[n / 4]}+(\tau+1) h / 2, t=y_{[n / 4]}+(\tau+1) h / 2$, and $\tau=\xi=2 / 3$.

\begin{tabular}{lccll}
\hline & $h$ extra & $h^{2}-$ extra & $h^{3}-$ extra & \\
\hline 16 & $-2.3988 e-01$ & & & \\
32 & $-1.1270 e-01$ & $1.4482 e-02$ & $6.8556 e-03$ & \\
64 & $-5.1968 e-02$ & $8.7623 e-03$ & $1.1491 e-03$ & $3.3393 e-04$ \\
128 & $-2.4458 e-02$ & $3.0524 e-03$ & $1.6570 e-04$ & $2.5214 e-05$ \\
256 & $-1.1785 e-02$ & $8.8738 e-04$ & $2.2231 e-05$ & $1.7349 e-06$ \\
512 & $-5.7733 e-03$ & $2.3852 e-04$ & $2.8785 e-06$ & $1.1385 e-07$ \\
1024 & $-2.8558 e-03$ & $6.1789 e-05$ & & \\
\hline
\end{tabular}

TABLE 8: A posterior error of the rectangle rule with $s=x_{[n / 4]}+(\tau+1) h / 2, t=y_{[n / 4]}+(\tau+1) h / 2$, and $\tau=\xi=2 / 3$.

\begin{tabular}{lcccc}
\hline & $h$-extra & $h^{2}-$ extra & $h^{3}-$ extra & \\
\hline 16 & & & & \\
32 & $-1.2718 e-01$ & & & \\
64 & $-6.0730 e-02$ & $1.9067 e-03$ & $1.4049 e-04$ & \\
128 & $-2.7510 e-02$ & $1.9033 e-03$ & $8.1521 e-04$ & $2.0581 e-05$ \\
256 & $-1.2673 e-02$ & $7.2168 e-04$ & $2.0496 e-05$ & $1.5653 e-06$ \\
512 & $-6.0118 e-03$ & $2.1629 e-04$ & $2.7647 e-06$ & $1.0807 e-07$ \\
1024 & $-2.9175 e-03$ & $5.8910 e-05$ & & \\
\hline
\end{tabular}

TABLE 9: Extrapolation errors of the rectangle rule with $t=x_{[n / 4]}+\left(\tau_{1}+1\right) h / 2, s=x_{[n / 4]}+\left(\tau_{2}+1\right) h / 2, u=x_{[n / 4]}+\left(\tau_{3}+1\right) h / 2, s, \tau_{1}=\tau_{2}=\tau_{3}=0$.

\begin{tabular}{lcccr}
\hline & $h-$ extra & $h^{2}-$ extra & $h^{3}-$ extra & \\
\hline 8 & $-2.2044 e-01$ & & & \\
16 & $-1.3967 e-01$ & $-5.8905 e-02$ & & \\
32 & $-7.9385 e-02$ & $-1.9096 e-02$ & $-5.8267 e-03$ & $-1.5932 e-04$ \\
64 & $-4.2405 e-02$ & $-5.4249 e-03$ & $-8.6774 e-04$ & $-8.7182 e-06$ \\
128 & $-2.1924 e-02$ & $-1.4433 e-03$ & $-1.1610 e-04$ & $-4.4454 e-07$ \\
256 & $-1.1148 e-02$ & $-3.7200 e-04$ & $-1.4901 e-05$ & \\
\hline
\end{tabular}

TABLE 10: A posterior error of the rectangle rule with $t=x_{[n / 4]}+\left(\tau_{1}+1\right) h / 2, s=x_{[n / 4]}+\left(\tau_{2}+1\right) h / 2, u=x_{[n / 4]}+\left(\tau_{3}+1\right) h / 2, s, \tau_{1}=\tau_{2}=\tau_{3}=0$.

\begin{tabular}{lcccc}
\hline & $h-$ extra & $h^{2}-$ extra & $h^{3}-$ extra & \\
\hline 8 & & & & \\
16 & $-8.0769 e-02$ & & & \\
32 & $-6.0289 e-02$ & $-1.3270 e-02$ & $-7.0842 e-04$ & $-1.0040 e-05$ \\
64 & $-3.6980 e-02$ & $-4.5571 e-03$ & $-1.0738 e-04$ & $-5.5157 e-07$ \\
128 & $-2.0481 e-02$ & $-1.3272 e-03$ & $-1.4456 e-05$ & \\
256 & $-1.0776 e-02$ & $-3.5710 e-04$ & & \\
\hline
\end{tabular}

TABLE 11: Extrapolation errors of the rectangle rule with $t=x_{[n / 4]}+\left(\tau_{1}+1\right) h / 2, s=x_{[n / 4]}+\left(\tau_{2}+1\right) h / 2, u=x_{[n / 4]}+\left(\tau_{3}+1\right) h / 2, s$, $\tau_{1}=\tau_{2}=\tau_{3}=2 / 3$.

\begin{tabular}{lllll}
\hline & $h-$ extra & $h^{2}-$ extra & $h^{3}-$ extra & \\
\hline 8 & $-2.2044 e-01$ & & & \\
16 & $-1.3967 e-01$ & $-5.8905 e-02$ & & \\
32 & $-7.9385 e-02$ & $-1.9096 e-02$ & $-5.8267 e-03$ & $-1.5932 e-04$ \\
64 & $-4.2405 e-02$ & $-5.4249 e-03$ & $-8.6774 e-04$ & $-8.7182 e-06$ \\
128 & $-2.1924 e-02$ & $-1.4433 e-03$ & $-1.1610 e-04$ & $-4.4454 e-07$ \\
256 & $-1.1148 e-02$ & $-3.7200 e-04$ & $-1.4901 e-05$ & \\
\hline
\end{tabular}

TABLE 12: A posterior error of the rectangle rule with $t=x_{[n / 4]}+\left(\tau_{1}+1\right) h / 2, s=x_{[n / 4]}+\left(\tau_{2}+1\right) h / 2, u=x_{[n / 4]}+\left(\tau_{3}+1\right) h / 2, s$, $\tau_{1}=\tau_{2}=\tau_{3}=2 / 3$.

\begin{tabular}{lcccc}
\hline & $h-$ extra & $h^{2}-$ extra & $h^{3}-$ extra & \\
\hline 8 & & & & \\
16 & $-8.0769 e-02$ & & & \\
32 & $-6.0289 e-02$ & $-1.3270 e-02$ & $-7.0842 e-04$ & $-1.0040 e-05$ \\
64 & $-3.6980 e-02$ & $-4.5571 e-03$ & $-1.0738 e-04$ & $-5.5157 e-07$ \\
128 & $-2.0481 e-02$ & $-1.3272 e-03$ & $-1.4456 e-05$ & \\
256 & $-1.0776 e-02$ & $-3.5710 e-04$ & & \\
\hline
\end{tabular}


the rectangle rule extrapolation convergence rate of the rectangle rule is $O(h), O\left(h^{2}\right), O\left(h^{3}\right), \ldots$, with $\tau 1=\tau 2=\tau 3=0$, which agrees with our Theorem 4 .

Tables 11 and 12 show extrapolation errors of the rectangle rule with the singular points $t=x_{[n / 4]}+\left(\tau_{1}+1\right) h / 2, s=$ $x_{[n / 4]}+\left(\tau_{2}+1\right) h / 2$, and $u=x_{[n / 4]}+\left(\tau_{3}+1\right) h / 2$; the convergence rate of the rectangle rule is $O(h), O\left(h^{2}\right), O\left(h^{3}\right), \ldots$, for the general convergence rate, while a posterior error of the rectangle rule extrapolation convergence rate of the rectangle rule is $O(h), O\left(h^{2}\right), O\left(h^{3}\right), \ldots$, with $\tau_{1}=\tau_{2}=\tau_{3}=2 / 3$, which agrees with our Theorem 4 .

\section{Conclusion}

In this paper, we have shown, both theoretically and numerically, that the main part of error functional of the rectangle rule has the asymptotic expansion (18). Numerical experiment has shown that the special function $a_{i, j}(\tau, \xi)$ has a big influence on the convergence rate. Moreover, by the extrapolation method, we not only obtain a high order of accuracy but also derive a posteriori error estimate conveniently. In fact, it is not difficult to extend our results to arbitrary (multidimensional) Cauchy principal value integral and the extrapolation methods can be similarly obtained.

\section{Data Availability}

The data used to support the findings of this study are included within the article.

\section{Conflicts of Interest}

The authors declare that they have no conflicts of interest.

\section{Acknowledgments}

The work of Jin Li was supported by the Natural Science Foundation of Shandong Province (Grant no. ZR2016JL006), Natural Science Foundation of Hebei Province (Grant no. A2019209533), National Natural Science Foundation of China (Grant no. 11471195 and 11771398), and China Postdoctoral Science Foundation (Grant no. 2015T80703).

\section{References}

[1] G. Monegato, "Convergence of product formulas for the numerical evaluation of certain two-dimensional Cauchy principal value integrals," Numerische Mathematik, vol. 43, no. 2, pp. 161-173, 1984.

[2] J. T. Chen and H.-K. Hong, "Review of dual boundary element methods with emphasis on hypersingular integrals and divergent series," Applied Mechanics Reviews, vol. 52, no. 1, pp. 17-33, 1999.

[3] G. Criscuolo and G. Mastroianni, "Convergence of Gauss type product formulas for the evaluation of two-dimensional Cauchy principal value integrals," $B I T$, vol. 27 , no. 1, pp. 72-84, 1987.

[4] Z. K. Mastroianni, N. M. A. Nik Long, and M. Abdulkawi, "Approximate solution of singular integral equations of the first kind with Cauchy kernel," Applied Mathematics Letters, vol. 22, no. 5, pp. 651-657, 2009.

[5] I. K. Lifanov, Singular Integral Equations and Discrete Vortices, VSP, Utrecht, Netherlands, 1996.

[6] D. H. Yu, Natural Boundary Integrals Method and Its Applications, Kluwer Academic Publishers, Alphen aan den Rijn, Netherlands, 2002.

[7] P. Keller and I. Wróbel, "Computing Cauchy principal value integrals using a standard adaptive quadrature," Journal of Computational and Applied Mathematics, vol. 294, pp. 323$341,2016$.

[8] Z. K. Eshkuvatov, N. M. A. Nik Long, and M. Abdulkawi, "Numerical evaluation for Cauchy type singular integrals on the interval," Journal of Computational and Applied Mathematics, vol. 233, no. 8, pp. 1995-2001, 2010.

[9] N. I. Ioakimidis, "On the uniform convergence of Gaussian quadrature rules for Cauchy principal value integrals and their derivatives," Mathematics of Computation, vol. 44, no. 169 , p. 191, 1985.

[10] P. J. Davis and P. Rabinowitz, Methods of Numerical Integration, Academic Press, New York, USA, 2nd edition, 1984.

[11] J. Li and D. H. Yu, "Error expansion of classical trapezoidal rule for computing Cauchy principal value integral," CMES-Computer Modeling in Engineering \& Sciences, vol. 93, pp. 47-67, 2013.

[12] J. Li, J. E. Yang, and D. Yu, "Error expansion of classical midpoint rectangle rule for computing Cauchy principal value integrals on an interval," International Journal of Computer Mathematics, vol. 91, no. 10, pp. 2294-2306, 2014.

[13] J. Li, H. Rui, and D. Yu, "Trapezoidal rule for computing supersingular integral on a circle," Journal of Scientific Computing, vol. 66, no. 2, pp. 740-760, 2016.

[14] P. Koler, "On the error of quadrature formulae for Cauchy principal value integrals based on piecewise interpolation," The Journal of Approximation Theory and its Applications, vol. 13, pp. 58-69, 1997.

[15] D. H. Yu, "The approximate computation of hypersingular integrals on interval," Numerical Mathematics: A Journal of Chinese Universities (English Series), vol. 1, pp. 114-127, 1992.

[16] J. Wu and W. Sun, "The superconvergence of Newton-Cotes rules for the Hadamard finite-part integral on an interval," Numerische Mathematik, vol. 109, no. 1, pp. 143-165, 2008.

[17] J. M. Wu and D. H. Yu, "The approximate computation of hypersingular integrals on interval," Chinese Journal of $\mathrm{Nu}$ merical Mathematics and Applications, vol. 21, pp. 25-33, 1999.

[18] X. Zhang, J. Wu, and D. Yu, "Superconvergence of the composite Simpson's rule for a certain finite-part integral and its applications," Journal of Computational and Applied Mathematics, vol. 223, no. 2, pp. 598-613, 2009.

[19] X. Zhang, J. Wu, and D. Yu, "The superconvergence of composite Newton-Cotes rules for Hadamard finite-part integral on a circle," Computing, vol. 85, no. 3, pp. 219-244, 2009.

[20] J. Li and D. H. Yu, "The superconvergence of certain twodimensional Cauchy principal value integrals," CMES-Computer Modeling in Engineering \& Sciences, vol. 71, pp. 331-346, 2011.

[21] J. Li and D. H. Yu, "The superconvergence of certain twodimensional hilbert singular integrals," CMES-Computer Modeling in Engineering \& Sciences, vol. 82, pp. 233-252, 2011.

[22] J. Li and D. H. Yu, "Collocation methods to solve certain Hilbert integral equation with middle rectangle rule," 
CMES-Computer Modeling in Engineering \& Sciences, vol. 102, no. 2, pp. 103-126, 2014.

[23] G. Monegato, "The numerical evaluation of a 2-D Cauchy principal value integral arising in boundary integral equation methods," Mathematics of Computation, vol. 62, no. 206, pp. 765-777, 1994.

[24] N. I. Ioakimidis, "Two-dimensional principal value hypersingular integrals for crack problems in three-dimensional elasticity," Acta Mechanica, vol. 82, no. 1-2, pp. 129-134, 1990.

[25] J. Li and H. Rui, "Error expansion of trapezoidal rule for certain two-dimensional Cauchy principal value integrals," Computers \& Mathematics with Applications, vol. 74, no. 10, pp. 2608-2637, 2017.

[26] J. Li, Q. Zhao, and H. Huang, "Error expansion of piecewise constant interpolation rule for certain two-dimensional Cauchy principal value integrals," Computers \& Mathematics with Applications, vol. 72, no. 9, pp. 2119-2142, 2016.

[27] C. Dagnino and A. P. Orsi, "On the evaluation of certain twodimensional singular integrals with Cauchy kernels," Numerische Mathematik, vol. 46, no. 1, pp. 121-130, 1985.

[28] M. M. Nayak, M. Acharya, and B. P. Acharya, "Numerical evaluation of two-dimensional Cauchy principal value integrals," Applied Mathematics and Computation, vol. 225, pp. 807-810, 2013.

[29] R. Piessens, "Numerical evaluation of Cauchy principal values of integrals," BIT, vol. 10, no. 4, pp. 476-480, 1970.

[30] S. M. Belotserkovsky, I. K. Lifanov, and V. A. Khokhryakov, Method of Discrete Vortices English Edition Editor, G. P. Cherepanov, CRC Press, Boca Raton, FL, USA, 1993.

[31] J. N. Lyness, “The euler maclaurin expansion for the Cauchy principal value integral," Numerische Mathematik, vol. 46, no. 4, pp. 611-622, 1985.

[32] J. N. Lyness, "Finite-part integrals and the Euler-Maclaurin expansion," in Approximation and Computation: ISNM International Series of Numerical Mathematics, R. V. M. Zahar, Ed., vol. 119, pp. 397-407, Springer, Berlin, Germany, 1994.

[33] J. Li, J. M. Wu, and D. H. Yu, "Generalized extrapolation for computation of hypersingular integrals in boundary element methods," CMES-Computer Modeling in Engineering \& Sciences, vol. 42, no. 2, pp. 151-175, 2009.

[34] J. Li, X. Zhang, and D. Yu, "Extrapolation methods to compute hypersingular integral in boundary element methods," Science China Mathematics, vol. 56, no. 8, pp. 1647-1660, 2013.

[35] J. Huang, Z. Wang, and R. Zhu, "Asymptotic error expansions for hypersingular integrals," Advances in Computational Mathematics, vol. 38, no. 2, pp. 257-279, 2013.

[36] A. Sidi, "Compact numerical quadrature formulas for hypersingular integrals and integral equations," Journal of Scientific Computing, vol. 54, pp. 145-176, 2013.

[37] L. C. Andrews, Special Functions of Mathematics for Engineers, McGraw-Hill, New York, NY, USA, 2nd edition, 1992.

[38] A. Sidi, Practical Extrapolation Methods Theory and Applications, Cambridge University Press, Cambridge, UK, 2003. 